\title{
Hip precautions after hemiarthroplasty: what is happening in the UK and at what cost?
}

\author{
R Fox ${ }^{1}$, R Halliday ${ }^{1}$, S Barnfield ${ }^{1}$, J Roxburgh $^{1}$, J Dunford $^{2}$, TJS Chesser ${ }^{1}$ \\ Departments of ${ }^{1}$ Trauma and Orthopaedics and ${ }^{2}$ Occupational Therapy, Frenchay Hospital, \\ Bristol, UK
}

\section{ABSTRACT}

INTRODUCTION The aim of this study was to observe current practice of the use of hip precautions following hemiarthroplasty for hip fractures in England and to audit the cost of hip precautions in this patient group.

METHODS A telephone review was undertaken of all units identified by the National Hip Fracture Database as receiving centres for hip fractures across England to ascertain current practice in the use of hip precautions. A prospective audit of occupational therapy (OT) practice including the cost of equipment provision and OT time was carried out locally. RESULTS All 174 units in England were successfully contacted. Practice varied between centres but hip precautions were in use at $78 \%$ of centres. Prior to stopping hip precautions at the local hospital, we audited the costs associated with their use. Mean equipment costs per patient decreased by $£ 12$ (from $£ 49$ to $£ 37$, range: $£ 0-£ 83$ ) and mean OT time per patient decreased by 1.5 hours (from 8 hours to 6.5 hours, range: $1-22$ hours) following removal of hip precaution guidelines. A mean of 0.25 days' discharge delay (range: $0-4$ days) due to equipment provision was identified prior to removing hip precautions with no discharge delay following.

CONCLUSIONS This study has highlighted the variation in practice across the country and inconsistency with the advice published by the British Orthopaedic Association and British Geriatrics Society in the 'Blue Book' (The Care of Patients with Fragility Fracture). Hip precautions are unnecessary after hemiarthroplasty, cost money both in therapist time and equipment provision and increase the length of hospital stay. Nevertheless, they continue to be used by three-quarters of trauma hospitals in England.

\section{KEYWORDS}

Femoral neck fractures - Hip fractures - Dislocations - Occupational therapy - Physical therapy Rehabilitation

Accepted May 192011

\section{CORRESPONDENCE TO}

Rebecca Fox, Research Physiotherapist, Department of Trauma and Orthopaedics, Frenchay Hospital, Frenchay Park Road, Bristol BS16 1LE, UK

T: +44 (0)117 340 2801; E: rebecca.fox@nbt.nhs.uk

The treatment of hip fractures remains a challenge to any health community and there have been many recent publications making suggestions to improve care. It has also been increasingly recognised that hip precautions after hemiarthroplasty are unnecessary. The combined publication on the treatment of the elderly with fragility fractures by the British Orthopaedic Association (BOA) and British Geriatric Society (BGS) states: 'for a hemiarthroplasty introduced via an antero-lateral approach [hip precautions] should no longer be necessary'. ${ }^{1}$ Hip precautions consist of patient education and equipment provision to reduce the risk of hip dislocation by enforcing restrictions of range of movement and limiting functional tasks. ${ }^{2}$ This utilises valuable therapist time, requires specialised equipment and can potentially delay discharge while it is being arranged.

The aim of this study was to observe the current practice of the use of hip precautions following hemiarthroplasty in trauma-receiving hospitals in England. A secondary aim was to audit the cost of hip precautions in our local hospital.

\section{Methods}

Trauma units treating hip fractures across England were identified via the National Hip Fracture Database. A physiotherapist or an occupational therapist from each unit was asked whether hip precautions were used with patients undergoing treatment with hemiarthroplasty. If hip precautions were used, then further details on the length of time advised, what restrictions were implemented and the equipment provided were asked.

To establish the cost of following hip precautions within our hospital there was a six-month evaluation of occupational therapy activity with hemiarthroplasty patients, admitted from and discharged back to their own homes. A standard proforma was used by each occupational therapist that recorded equipment issued, time spent with each patient and any delay in discharge due to equipment delivery. Hip precautions in the local unit were then stopped in accordance with BOA/BGS guidelines. ${ }^{1}$ After a one-month period with no hip precautions, the audit of occupational therapy input was repeated. 


\section{Results}

In England 184 units were identified by the National Hip Fracture Database in 2008. Of these, 10 were excluded either due to a merger with another unit or because the unit did not carry out any orthopaedic trauma surgery. All remaining 174 units were successfully contacted.

Of the 174 units treating hip fractures, 135 units $(78 \%)$ advised patients to follow hip precautions following hemiarthroplasty. In six (4\%) of these units, hip precautions were recommended by occupational therapists only and not by the physiotherapists. The majority of units (53\%) recommended patients continued precautions for three months and a further $28 \%$ of units recommended six weeks (range: six weeks to indefinite).

The provision of equipment to enable patients to comply with the hip precautions varied from dressing aids to raised seating. Some respondents stated that equipment was provided to all patients routinely, others that it was dependent on the occupational therapist's assessment of each patient. Several units commented that they provided some equipment but advised patients on the purchase of other items.

In the local unit studied, occupational therapists spent a mean of 8 hours (range: 1-22 hours) with each patient and provided a mean of $£ 49$ (range: $£ 0-\mathfrak{£ 8 3}$ ) worth of equipment. After hip precautions were stopped, time spent with patients reduced by a mean of 1.5 hours (range: $0-18$ hours) per patient and the mean cost of equipment provision reduced by $£ 12$ to a mean value of $£ 37$ (range: $£ 0-£ 100$ ) per patient. Delays to patient discharge due to equipment provision ranged from 0 to 4 days. This delay was eradicated following the removal of hip precautions (a mean reduction of 0.25 days per patient). There was no increase in dislocation rate in the year after stopping precautions $(<1 \%)$.

\section{Discussion}

There remains controversy over the use of hip precautions after hemiarthroplasty in patients undergoing treatment for intracapsular hip fractures. Although dislocation rates as high as $6 \%$ have been reported, ${ }^{3}$ most studies quote rates of between $1 \%$ and $2 \%{ }^{4,5}$ Reasons precautions are not required include the use of large femoral head components (usually $>42 \mathrm{~mm}$ in diameter) and an anterolateral approach. ${ }^{5,6,7}$ The prevalence of cognitive impairment and post-operative delirium causes difficulty in implementation of hip precautions in $30 \%$ of this population. ${ }^{8}$ The use of hip precautions can delay mobilisation, rehabilitation and discharge and can increase patient anxiety. ${ }^{9}$

The potential cost of this unnecessary practice to local hospitals and the National Health Service as a whole is concerning. This study only considered practice in England; however, if the figures are extrapolated to all patients in the UK (70,000 patients a year, $45 \%$ receive a hemiarthroplasty), the additional cost of employing hip precautions could involve an extra 3,400 bed days and approximately $£ 165,000$ worth of equipment per year.

Despite advice from the BOA and $\mathrm{BGS}^{1}$ being widely disseminated, $78 \%$ of units are still employing precautions. Our findings correlate with the findings of McQueen et al who performed a voluntary postal survey of occupational therapists with a response rate of $50 \%$, of which $70 \%$ continued to use hip precautions. ${ }^{2}$ The reasons stated for their continued use included historical concern and consultant choice. This may be due to ignorance of the continued practice by the consultant body, as was in our case.

Only one member of staff from each unit was surveyed (95\% physiotherapists), which cannot be guaranteed to be representative of the practice of the whole multidisciplinary team. McQueen et al reported varying practice even within the same team of occupational therapists. ${ }^{2}$ Although the financial implications of hip precautions reported are specific to the study centre, if extrapolated, the findings are significant considering the size and anticipated growth of the patient population.

\section{Conclusions}

This study has confirmed that the majority of orthopaedic trauma units throughout the UK continue to use hip precautions for their patients following hemiarthroplasty. This is despite national recommendations given by the BOA/BGS and at significant cost. Our findings reaffirm the need for good communication and continuing evaluation of practice within local multidisciplinary teams to achieve optimum and timely care in this patient group. We urge those who have responsibility for these patients to review their own practice. Hip precautions are unnecessary after hemiarthroplasty, cost money both in therapist time and equipment provision, increase the length of hospital stay and yet continue to be used by threequarters of trauma hospitals in England.

\section{References}

1. British Orthopaedic Association, British Geriatrics Society. The Care of Patients with Fragility Fracture. London: BOA; 2007.

2. McQueen J, Nivison C, Ballance $F$ et al. Hip precautions following hemiarthroplasty: a UK study of occupational therapists. Int J Ther Rehabil 2009; 16: 147-153.

3. Enocson A, Tidermark J, Tornkvist $H$, Lapidus LJ. Dislocation of hemiarthroplasty after femoral neck fracture: better outcome after the anterolateral approach in a prospective cohort study on 739 consecutive hips. Acta Orthop 2008; 79: 211-217.

4. Barnes CL, Berry DJ, Sledge CB. Dislocation after bipolar hemiarthroplasty of the hip. J Arthroplasty 1995; 10: 667-669.

5. Sierra RJ, Schleck CD, Cabanela ME. Dislocation of bipolar hemiarthroplasty: rate, contributing factors, and outcome. Clin Orthop Relat Res 2006; 442: 230-238.

6. Keene GS, Parker MJ. Hemiarthroplasty of the hip - the anterior or posterior approach? A comparison of surgical approaches. Injury 1993; 24: 611-613.

7. Bush JB, Wilson MR. Dislocation after hip hemiarthroplasty: anterior versus posterior capsular approach. Orthopedics 2007; 30: 138-144.

8. Givens JL, Sanft TB, Marcantonio ER. Functional recovery after hip fracture: the combined effects of depressive symptoms, cognitive impairment, and delirium. J Am Geriatr Soc 2008; 56: 1,075-1,079.

9. Kondo $A$, Kanda $K$, Isokawa $Y$ et al. The relationship in hip fracture patients of post-operative days to initial rehabilitation activities and their ambulatory ability on post-operative day 20. J Orthop Nurs 2005; 9: 146-155. 